\title{
Has Mayaro virus always existed or is it new?
}

Juan Vargas

Mayaro virus has always been one of the significant challenges for biomedical science, and efforts are aimed at intervening factors preventing viruses from spreading. This letter reviews recent research on emerging zoonotic viruses in Latin America, focusing on Mayaro virus as a new threat. It also considers vector and clinical characteristics and similarities with other arboviruses (arthropod borne), such as Zika virus, dengue virus, and chikungunya virus [1].

The diseases caused by arboviruses are very serious worldwide, and since their surveillance and prevention requires monitoring of their vectors, their control is difficult, and their expansion to any tropical or subtropical country is almost impossible to avoid.

At the same time, suitable eco-epidemiological conditions of tropical and subtropical regions have allowed the persistence of arboviruses by providing an ideal blend in terms of vector usage and specificity as well as an ample host range [2]. Newly introduced arboviruses in the New World seem to be smoothly transitioning from their epidemic effervescence into endemic levels of transmission, revealing an increased potential for adaptation [2].

Although these risk factors are taken into account, research indicates that the relationship between these factors and the spread of arboviruses in South America is necessary, but this cannot be the only geographic region affected by these factors.

Mayaro fever is a zoonosis caused by Mayaro virus, an arbovirus of the genus Alphavirus of the family Togaviridae, endemic to moist forests in the tropical South American region and transmitted by Haemagogus mosquitoes [3]. The Aedes aegypti mosquito can also serve as a competent vector; therefore the potential for Mayaro virus to emerge as a global pathogen is worrisome [1].

The reason for the new hypotheses about Aedes aegypti is that in May 2016 Mayaro virus was found in an 8-year-old boy with an acute febrile illness in a "semirural" area (Gressier-Leogane) approximately 20 miles west of Port-au-Prince, Haiti. The fact that Mayaro virus infection was found in someone from a nonforest area and occurred in the context of a coinfection with dengue virus suggests that Aedes aegypti may have been the mosquito vector responsible for transmission [4].

It has also been observed that Haiti is not a habitat for wild nonhuman primates, which might suggest a different reservoir or human-to-human transmission by Aedes mosquitoes [4]. However, it remains unknown whether Aedes aegypti or other Aedes mosquitoes are efficient vectors for Mayaro virus. Also, there is a single report on the isolation of Mayaro virus from birds, but the role of birds in virus transmission remains unknown [4].

Also, Mayaro virus infection constitutes a double risk for health and public health entities because it shares clinical signs and symptoms that are confused with dengue, chikungunya, and Zika virus disease, and in particular chikungunya with prolonged arthralgia [3]. From
CORRESPONDING AUTHOR: Juan Vargas

Simón Bolivar University, Medical Program

E-mail: vargassebastian152@ gmail.com

Orcid: 0000-0002-6698-5864

Received 16 October 2017; Accepted 5 January 2018 
national experiences around the world, especially in places that meet the ecological and environmental conditions conducive to the distribution of vectors, there could be cases mistakenly classified initially as dengue, chikungunya, or Zika virus disease, suggesting the presence of other infectious agents not previously considered, including Mayaro virus [3].

One of the largest outbreaks of Mayaro virus was reported in 2015 by Scott Weaver in a rural village in northwestern Venezuela, where 77 cases were found, including 19 confirmed seropositive individuals [4]. These findings, together with the first report of Mayaro virus infection in an HIV-infected patient, raised concerns that Mayaro virus could become a major emerging pathogen in South America, leading to early attempts to develop vaccines [5].

Could Mayaro virus infection become the "next chikungunya" in the Americas? Mario Luis García de Figueiredo and Luiz Tadeu Moraes Figueiredo of the University of São Paulo in Brazil have suggested that both alphaviruses may "mutate and / or adapt to new zoonotic cycles and thus acquire greater potential for emergence" to cause significant epidemics [6]. This is an important hypothesis that requires research but will be difficult to investigate [4].

Some authors suggest that the control and prevention of arboviruses should focus on the control of vectors, to avoid the emergence of new arboviruses or new mutations of existing serotypes of arboviruses and to avoid the destruction of efforts in the treatment of these diseases [1]. Although there have been few reported cases of infection with Mayaro virus, we must not lower our guard as it has the potential to emerge as a global pathogen [3].

\section{References}

1. Arredondo-García JL, Méndez-Herrera A, Medina-Cortina H Arbovirus en Latinoamérica. Acta Pediatr Mex 2016;37(2):111-31.

2. Paniz-Mondolfi AE, Rodriguez-Morales AJ, Blohm G, Marquez M, Villamil-Gomez WE. ChikDenMaZika syndrome: the challenge of diagnosing arboviral infections in the midst of concurrent epidemics. Ann Clin Microbiol Antimicrob 2016;15(1):42.

3. Cárdenas Marrufo MF, Pech Sosa N, Arias León JJ. Mayaro: enfermedad emergente al acecho. Cien Hum Salud 2016:3(3):32-40.

4. Auguste AJ, Liria J, Forrester NL, Giambalvo D, Moncada M, Long $\mathrm{KC}$, et al. Evolutionary and ecological characterization of Mayaro virus strains isolated during an outbreak, Venezuela, 2010. Emerg Infect Dis 2015;21(10):1742-50.

5. Hotez PJ, Murray KO. Dengue, West Nile virus, chikungunya, Zika-and now Mayaro? PLoS Negl Trop Dis 2017:11(8):0005462.

6. De Figueiredo MLG, Figueiredo LTM. Emerging alphaviruses in the Americas: Chikungunya and Mayaro. Rev Soc Bras Med Trop. 2014;47(6):677-83. 